\title{
Natural Compound Shikonin Induces Apoptosis and Attenuates Epithelial to Mesenchymal Transition in Radiation-Resistant Human Colon Cancer Cells
}

\author{
Kristina Shilnikova, Mei Jing Piao, Kyoung Ah Kang, Pincha Devage Sameera Madushan Fernando, \\ Herath Mudiyanselage Udari Lakmini Herath, Suk Ju Cho* and Jin Won Hyun* \\ College of Medicine, Jeju National University, Jeju 63243, Republic of Korea
}

\begin{abstract}
Radiation resistance represents an imperative obstacle in the treatment of patients with colorectal cancer, which remains difficult to overcome. Here, we explored the anti-proliferative and migration-inhibiting properties of the natural product shikonin on a radiation-resistant human colon carcinoma cell line (SNU-C5RR). Shikonin reduced the viability of these cells in a dose-dependent manner; $38 \mu \mathrm{M}$ of shikonin was determined as the half-maximal inhibitory concentration. Shikonin induced apoptotic cell death, as demonstrated by increased apoptotic body formation and the number of TUNEL-positive cells. Moreover, shikonin enhanced mitochondrial membrane depolarization and Bax expression and also decreased Bcl-2 expression with translocation of cytochrome c from mitochondria into the cytosol. In addition, shikonin activated mitogen-activated protein kinases, and their specific inhibitors reduced the cytotoxic effects of shikonin. Additionally, shikonin decreased the migration of SNU-C5RR cells via the upregulation of $\mathrm{E}$-cadherin and downregulation of $\mathrm{N}$-cadherin. Taken together, these results suggest that shikonin induces mitochondria-mediated apoptosis and attenuates epithelial-mesenchymal transition in SNU-C5RR cells.
\end{abstract}

Key Words: Shikonin, Radiation resistance, Human colon cancer, Epithelial to mesenchymal transition, Mitochondria-mediated apoptosis, Mitogen-activated protein kinases

\section{INTRODUCTION}

Recent research reports, based on the analysis of morbidity and mortality databases from 39 countries, indicated that the incidence of colorectal cancer continues to increase in young people in countries with a high human development index (Wong et al., 2021). Republic of Korea had the second highest incidence of colorectal cancer in 2018 (Khil et al., 2021). Even after surgical intervention, some microscopic tumor cells may remain, which have the potential to invade the surrounding organs. Radiation therapy is commonly used to eliminate the remaining cells, which is termed adjuvant therapy (Baskar et al., 2012). Unfortunately, many clinical cases are associated with the development of radioresistance, which is difficult to overcome (Jin et al., 2016). Consequently, there is a great need for new chemotherapeutic agents with high anticancer activity to eradicate radioresistant cancer cells.

Recently, the scientific community has started to pay more attention to natural bioactive compounds and their anticancer properties. Shikonin is one of these naturally occurring compounds. Shikonin is extracted from Lithospermum erythrorhizon, Onosma paniculata, and Arnebia euchroma. Shikonin exerts cytotoxic effects by inhibiting protein tyrosine kinase and DNA topoisomerases, which play a crucial role in cancer cell DNA regulation, and decreases the expression of tumor necrosis factor receptor-associated protein 1. In addition, shikonin increases p53 expression and inhibits glycolysis by suppressing the pyruvate kinase M2 (Andújar et al., 2013; Han et al., 2015). Recently, the apoptosis-inducing effect of shikonin was shown in human stomach cancer cells, colon cancer cells, lung cancer cells, promyelocytic leukemia cells, medullary thyroid carcinoma cells, and cervical cancer cells (Lu et al., 2015; He et al., 2016; Jeung et al., 2016; Ko et al., 2016; Trivedi et al., 2016; Wei et al., 2016).

Most colon adenocarcinomas are silent tumors. This means slow growth and no obvious symptoms until the tumor reaches

\section{Open Access https://doi.org/10.4062/biomolther.2021.088}

This is an Open Access article distributed under the terms of the Creative Commons Attribution Non-Commercial License (http://creativecommons.org/licenses/by-nc/4.0/) which permits unrestricted non-commercial use, distribution, and reproduction in any medium, provided the original work is properly cited.
Received May 7, 2021 Revised Jun 7, 2021 Accepted Jun 8, 2021 Published Online Jul 29, 2021

\section{*Corresponding Authors}

E-mail: sukjucho@gmail.com (Cho SJ), jinwonh@jejunu.ac.kr (Hyun JW) Tel: +82-64-717-2062 (Cho SJ), +82-64-754-3838 (Hyun JW) Fax: +82-504-226-8173 (Cho SJ), +82-64-702-2687 (Hyun JW) 
a significant size. Then it becomes invasive and causes metastasis (Ameli and Khalily, 2016). This alteration is related to the epithelial to mesenchymal transition (EMT), the transformation of epithelial cells with adhesive properties that are in close contact with each other into high mobility mesenchymal cells, allowing penetration into surrounding tissues and metastasis (Zhu et al., 2013). The relationship between EMT and metastasis in colon cancer has been proven in many studies (Todosi et al., 2012; Zhu et al., 2013; Matějka et al., 2017). Upregulation of EMT inducers can promote EMT, leading to increased invasiveness and metastasis of colorectal cancer (Vu and Datta, 2017). These inducers can downregulate Ecadherin and upregulate $\mathrm{N}$-cadherin and vimentin through modulating EMT-related signaling pathways, such as WNT/ $\beta$ catenin and TGF- $\beta$, and EMT transcription factors, such as zinc finger E-box binding homeobox 1 (ZEB1) and ZEB2 (Vu and Datta, 2017). Therefore, prevention of EMT is an important step in anticancer therapy (Voon et al., 2017).

Here, we aimed to estimate the apoptosis-inducing and EMT-inhibiting properties of shikonin in radiation-resistant coIon cancer cells (SNU-C5RR).

\section{MATERIALS AND METHODS}

\section{Reagents}

Shikonin (5,8-dihydroxy-2-(1-hydroxy-4-methylpent-3-enyl) naphthalene-1,4-dione, Fig. 1A) was obtained from Cayman Chemical Company (Ann Arbor, MI, USA). 3-(4,5-Dimethylthiazol-2-yl)-2,5-diphenyltetrazolium bromide (MTT), Hoechst 33342 , trypan blue solution, 5,5-dimethyl-1-pyrroline-N-oxide (DMPO), 2', 7'-dichlorodihydrofluorescein diacetate (DCF-DA), and anti-actin antibody were obtained from Sigma-Aldrich (St. Louis, MO, USA). 5,5',6,6'-Tetrachloro-1,1',3,3'-tetraethylbenzimidazolocarbocyanine iodide (JC-1) was obtained from Invitrogen (Thermo Fisher Scientific, Inc., Waltham, MA, USA). Anti-phospho-JNK, anti-JNK, anti-phospho-p38, and anti-caspase-3 antibodies along with SB203580 (p38 inhibitor), and U0126 (ERK inhibitor) were obtained from Cell Signaling Technology, Inc (Danvers, MA, USA). Anti-Bax, anti-Bcl-2, anti-caspase-9, anti-cytochrome c oxidase subunit
4 (COX4), anti-phospho-ERK, anti-ERK, anti-p38, anti-E-cadherin, and anti-N-cadherin antibodies were supplied by Santa Cruz Biotechnology, Inc (Dallas, TX, USA). The JNK inhibitor SP600125 was provided by Selleck Chemicals (Houston, TX, USA). All other chemicals and reagents used were of analytical grade.

\section{Cell culture}

Human colorectal adenocarcinoma (SNU-C5) and radiation-resistant (SNU-C5RR) colon cancer cell lines were incubated in a humidified $5 \% \mathrm{CO}_{2}$ atmosphere at $37^{\circ} \mathrm{C}$ in RPMI medium containing $10 \%$ heat-inactivated fetal bovine serum (Gibco; Thermo Fisher Scientific, Inc.), penicillin (100 U/mL), and streptomycin $(100 \mu \mathrm{g} / \mathrm{mL})$.

\section{Cell viability assay}

SNU-C5 and SNU-C5RR cells were treated with various concentration of shikonin and then incubated for $24 \mathrm{~h}$. In addition, SNU-C5RR cells were treated with shikonin, SB203580, SP600125, U0126, or their combinations, and then incubated for $24 \mathrm{~h}$. To evaluate the EMT in SNU-C5RR cells, cell viability was assessed after $48 \mathrm{~h}$ of shikonin treatment. After each treatment, exactly $125 \mu \mathrm{L}$ of MTT solution ( $2 \mathrm{mg} / \mathrm{mL}$ ) was added to each well. After maintaining the cells at $37^{\circ} \mathrm{C}$ until the formation of formazan crystals, the supernatant was removed by aspiration. Then, we dissolved the formed formazan crystals in dimethyl sulfoxide solution and read the relevant absorbance values at $540 \mathrm{~nm}$ with a scanning multi-well spectrophotometer (Shilnikova et al., 2018).

\section{Colony formation assay}

SNU-C5RR cells were cultured in $60 \mathrm{~mm}$ dishes, and 400 cells per dish were used as the optimum cell density. Cultured cells were incubated, and $38 \mu \mathrm{M}$ shikonin was added on day 7 of incubation. The cell culture medium was changed every 3 days. On day 11 of incubation, $70 \%$ ethanol was applied to fix the cells, which were then stained with $0.4 \%$ trypan blue until colonies were detectable. Colony formation was imaged and quantified using ImageJ software (version 1.47; National Institutes of Health, Bethesda, MD, USA).<smiles>CC(C)=CCC(O)C1=CC(=O)c2c(O)ccc(O)c2C1=O</smiles>

B

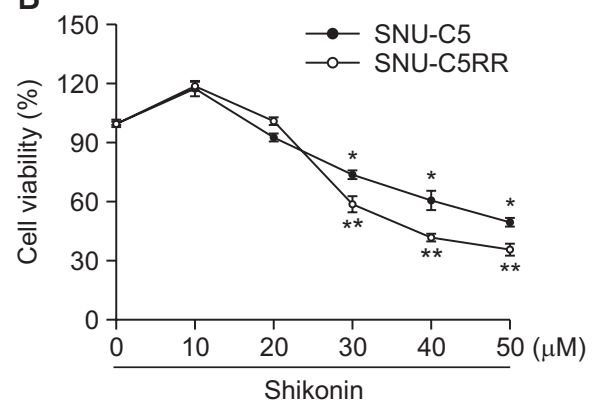

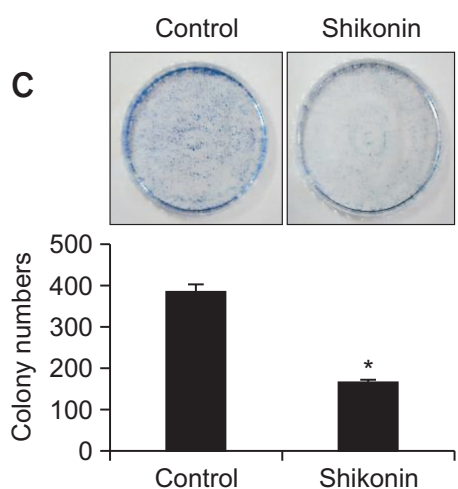

Fig. 1. Shikonin induces cell death in SNU-C5 and SNU-C5RR cells. (A) The chemical structure of shikonin [5,8-dihydroxy-2-(1-hydroxy4-methylpent-3-enyl)naphthalene-1,4-dione]. (B) SNU-C5 or SNU-C5RR cells were treated with 10, 20, 30, 40, or 50 $\mu \mathrm{M}$ shikonin for $24 \mathrm{~h}$. Cell viability was measured using the MTT assay. ${ }^{* * * *} p<0.05$ vs the control group in SNU-C5 and SNU-C5RR cells, respectively. (C) For the colony formation assay, SNU-C5RR cells were cultured for 11 days and treated with $38 \mu \mathrm{M}$ shikonin on day 7 . Colonies containing $>400$ cells were counted. * $p<0.05$ vs the control group. 


\section{Nuclear staining with Hoechst 33342}

After treating SNU-C5RR cells with $38 \mu \mathrm{M}$ shikonin or SP600125 along with SB203580, U0126, or a combination for $24 \mathrm{~h}$, Hoechst 33342 staining was performed, followed by incubation at $37^{\circ} \mathrm{C}$ for $10 \mathrm{~min}$. Then, cells were observed using a fluorescence microscope (Olympus Life Science, Tokyo, Japan).

\section{Terminal deoxynucleotidyl transferase-mediated dUTP nick end labeling (TUNEL) assay}

SNU-C5RR cells $\left(1 \times 10^{5}\right.$ cells $\left./ \mathrm{mL}\right)$ were cultured in chamber slides and maintained for $24 \mathrm{~h} .38 \mu \mathrm{M}$ Shikonin was added, and cells were incubated for $24 \mathrm{~h}$. The slides were then prepared following the manufacturer's instructions for the DeadEnd Colorimetric TUNEL system (Promega Corporation, Madison, WI, USA). Faramount aqueous mounting medium (Agilent Technologies, Inc., Santa Clara, CA, USA) was used to mount stained cells on microscope slides. The microscopic images were captured via confocal fluorescent microscopy using an appropriate Laser Scanning Microscope 5 PASCAL program (Carl Zeiss AG, Oberkochen, Germany).

\section{Single-cell gel electrophoresis (Comet assay)}

Cell pellets were suspended in $1 \%$ low-melting agarose (LMA), and the cell-containing suspension was evenly spread on a microscopic slide, which was pre-coated with $1 \%$ normal melting agarose. Slides were kept at $4^{\circ} \mathrm{C}$ to enhance the solidification of agarose and covered with $170 \mu \mathrm{L}$ of $0.5 \% \mathrm{LMA}$. Then, slides were dipped in lysis buffer $(2.5 \mathrm{M} \mathrm{NaCl}, 100 \mathrm{mM}$ $\mathrm{Na}_{2}$ EDTA, $1 \%$ Triton $\mathrm{X}-100,10 \mathrm{mM}$ Tris, and 1\% N-lauroyl sarcosinate, $\mathrm{pH} 10$ ) for $1 \mathrm{~h}$ in dark at $4^{\circ} \mathrm{C}$. Each slide was subjected to gel electrophoresis, which contained $\mathrm{NaOH}$ (300 $\mathrm{mM}$ ) and $\mathrm{Na}_{2}$ EDTA (10 mM, pH 10). Electrophoresis (300 mA, $25 \mathrm{~V}$ ) was conducted in the dark for $30 \mathrm{~min}$, resulting in DNA unwinding and the expression of alkali-labile damage. After electrophoresis, slides were immersed in neutralizing buffer (0.4 M Tris, $\mathrm{pH} 7.5$ ) and $70 \%$ ethanol. Immediately before detection, slides were stained with $40 \mu \mathrm{L}$ of ethidium bromide $(10 \mu \mathrm{g} / \mathrm{mL})$ and visualized under a fluorescence microscope (Olympus Life Science), and images were gathered using an image analyzer (Komet 5.5, Kinetic Imaging, Bromborough, UK). Exactly 25 cells per slide were used to quantify the comet tail length and total fluorescence percentage of the tails.

\section{Mitochondrial membrane potential $\left(\Delta_{\psi m}\right)$}

SNU-C5RR cells were incubated and treated with $38 \mu \mathrm{M}$ shikonin, then dyed with $\mathrm{JC}-1(10 \mu \mathrm{g} / \mathrm{mL})$ and maintained at $37^{\circ} \mathrm{C}$ to enhance the staining for another $15 \mathrm{~min}$. Microscopic slides were prepared using a mounting medium (DAKO, Carpinteria, CA, USA). The mitochondrial polarization and depolarization status were detected via confocal microscopy, which was implemented with the Laser Scanning Microscope 5 PASCAL software. In addition, data obtained via fluorescent microscopy were confirmed using a FACSCalibur flow cytometer (BD Biosciences, Franklin Lakes, NJ, USA).

\section{Western blotting}

SNU-C5RR cells were lysed using PRO-PREPTM protein extraction solution (Intron Biotechnology, Inc., Seongnam, Korea). Protein concentrations were quantified using a Bio-Rad protein assay reagent kit (Bio-Rad Laboratories, Inc., Hercules, CA, USA). Proteins were subjected to electrophoresis and transferred to nitrocellulose membranes (Bio-Rad Laboratories, Inc.). Nitrocellulose membranes were blocked in $2 \%$ fetal bovine serum (shaking), and incubated with primary antibodies (dilution, $1: 1,000$ ) overnight at $4^{\circ} \mathrm{C}$. The membranes were further incubated with secondary horseradish peroxidase-conjugated goat anti-rabbit immunoglobulin G (IgG) or goat antimouse IgG antibodies (Pierce; Thermo Fisher Scientific, Inc.; dilution, 1:5,000) for $1 \mathrm{~h}$ at room temperature. Corresponding protein bands were detected using an increased chemiluminescence western blotting detection kit (GE Healthcare Life Sciences, Little Chalfont, UK) and then exposed to X-ray films.

\section{Cell migration assay}

SNU-C5RR cells $\left(1 \times 10^{4}\right.$ cells $\left./ \mathrm{mL}\right)$ were seeded on $100 \mathrm{~mm}$ dishes, incubated for $24 \mathrm{~h}$, and then treated with $3 \mu \mathrm{M}$ shikonin. Then, scratch lines (diameter, $1.5 \mathrm{~mm}$ ) were established across the base of the culture dishes. The line areas were measured after 0 and $48 \mathrm{~h}$ using a fluorescence microscope (Olympus Life Science). The index of gap width was calculated as follows: (mean gap width, $\mathrm{mm}$ )/(mean gap width in control, mm).

\section{Detection of superoxide anion}

The xanthine/xanthine oxidase system was used to generate superoxide anions, which interacted with DMPO, and the produced $\mathrm{DMPO} / \cdot \mathrm{OOH}$ adducts were measured using an ESR spectrometer. The ESR spectrum was recorded 2.5 min after PBS ( $\mathrm{pH} 7.4$ ) was mixed with $0.02 \mathrm{~mL}$ of $3 \mathrm{M}$ DMPO, $5 \mathrm{mM}$ xanthine, $0.25 \mathrm{U}$ xanthine oxidase, and $3 \mu \mathrm{M}$ shikonin. ESR spectrometer parameters were as follows: Central magnetic field, $336.8 \mathrm{mT}$; power, $5.00 \mathrm{~mW}$; frequency, $9.4380 \mathrm{GHz}$; modulation width, $0.2 \mathrm{mT}$; amplitude, 1000; sweep width, 10 $\mathrm{mT}$; sweep time, $0.5 \mathrm{~min}$; time constant, $0.03 \mathrm{~s}$; temperature, $25^{\circ} \mathrm{C}$.

\section{Detection of hydroxyl radicals}

Hydroxyl radicals were generated through the Fenton reaction $\left(\mathrm{H}_{2} \mathrm{O}_{2}+\mathrm{FeSO}_{4}\right)$ and allowed to interact with DMPO. The resultant $\mathrm{DMPO} / \cdot \mathrm{OH}$ adducts were detected using an ESR spectrometer (Chae et al., 2011; Wu et al., 2012). The ESR spectrum was recorded 2.5 min after PBS ( $\mathrm{pH} 7.4)$ was mixed with $0.02 \mathrm{~mL}$ of $0.3 \mathrm{M} \mathrm{DMPO}, 10 \mathrm{mM} \mathrm{FeSO}_{4}, 10 \mathrm{mM} \mathrm{H}_{2} \mathrm{O}_{2}$, and $3 \mu \mathrm{M}$ shikonin. ESR spectrometer parameters were as follows: Central magnetic field, $336.8 \mathrm{mT}$; power, $1.00 \mathrm{~mW}$; frequency, $9.4380 \mathrm{GHz}$; modulation width, $0.2 \mathrm{mT}$; amplitude, 600; sweep width, $10 \mathrm{mT}$; sweep time, $0.5 \mathrm{~min}$; time constant, $0.03 \mathrm{~s}$; temperature, $25^{\circ} \mathrm{C}$.

\section{Intracellular reactive oxygen species (ROS) level}

SNU-C5RR cells were seeded on a coverslip-containing four-well plate at a density of $1.5 \times 10^{5}$ cells/well, and then cells were treated with $3 \mu \mathrm{M}$ shikonin. After $24 \mathrm{~h}$, representative cell groups were treated with DCF-DA (final concentration $20 \mu \mathrm{M}$ ) and incubated for $30 \mathrm{~min}$ at $37^{\circ} \mathrm{C}$. Stained cells were washed with PBS, and the coverslips were mounted onto microscope slide in a mounting medium (DAKO). Then, the slides were analyzed using a confocal microscope. Relevant images were gathered using the Laser Scanning Microscope 5 PASCAL software (Carl Zeiss, Jena, Germany). In addition, data obtained via fluorescent microscopy were confirmed using a FACS Calibur flow cytometer (BD Biosciences). 
A
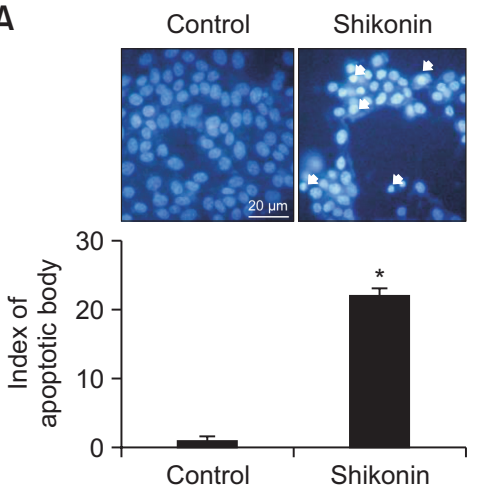

B

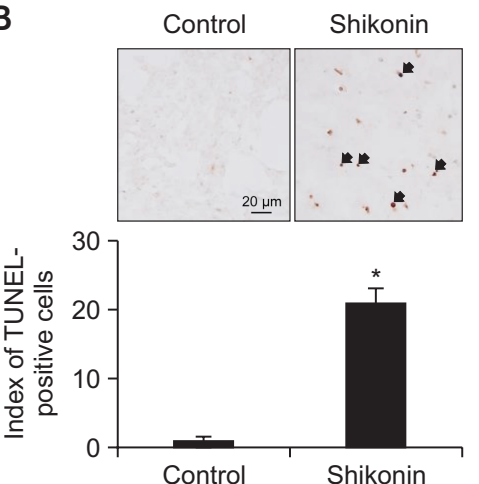

C
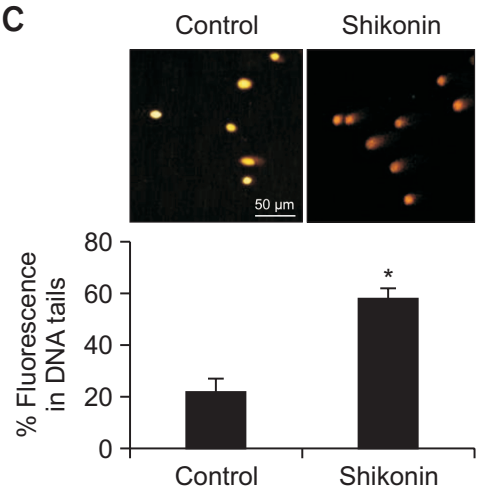

Fig. 2. Shikonin induces apoptosis in SNU-C5RR colon cancer cells. (A) Apoptotic bodies (arrows) were observed via fluorescence microscopy in SNU-C5RR cells stained with Hoechst 33342 and quantified. ${ }^{*} p<0.05$ vs the control group. (B) Apoptosis in SNU-C5RR cells was examined via the TUNEL assay after $24 \mathrm{~h}$ of $38 \mu \mathrm{M}$ shikonin treatment. TUNEL-positive cells are indicated by arrows. ${ }^{*} p<0.05$ vs the control group. (C) DNA damage was assessed using an alkaline comet assay. Representative images and the percentage of total DNA fluorescence in the comet tails are shown. ${ }^{*} p<0.05$ vs the control group.

\section{Statistical analysis}

All experiments were performed in triplicates. Values represent the mean \pm standard error of the mean. All data were evaluated via one-way analysis of variance with Tukey's posthoc test to analyze the differences. Differences were considered statistically significant at a $p$ value $<0.05$.

\section{RESULTS}

\section{Shikonin decreases cell viability in colon carcinoma cell lines}

Shikonin displayed an obvious dose-dependent toxic effect on both parental (SNU-C5) and radiation-resistant (SNUC5RR) human colon carcinoma cell lines (Fig. 1B). The IC50 was $52 \mu \mathrm{M}$ for SNU-C5 cells and $38 \mu \mathrm{M}$ for SNU-C5RR cells. In other words, both parental and radiation-resistant cell lines were strongly sensitive to shikonin. To further confirm this finding, we examined the colony-forming ability of SNU-C5RR cells (Fig. 1C). Shikonin (38 $\mu \mathrm{M})$ significantly decreased the number of colonies formed compared to that in the control group. Therefore, we used $38 \mu \mathrm{M}$ as the final concentration for further experiments to test the cytotoxic properties of shikonin in the SNU-C5RR cell line.

\section{Shikonin induces apoptosis in radiation-resistant colon carcinoma cells}

To determine whether the toxic effect of shikonin was related to apoptosis, we detected apoptotic body formation via TUNEL assay as well as the proportion of DNA condensation via Hoechst 33342 nuclear staining. Shikonin-treated cells possessed a higher number of apoptotic bodies and TUNELpositive cells compared to the control group (Fig. 2A, 2B). Damaged DNA was differentiated from normal DNA due to the influence of the electrophoretic field via comet assay, exhibiting the classic comet tail. Shikonin-treated cells demonstrated elongated nuclear tails and an enhanced DNA index in the tails (Fig. 2C).

\section{Shikonin initiates mitochondria-mediated apoptosis}

Mitochondria in control cells displayed red fluorescence upon staining with JC-1, which was visualized through $\Delta_{\psi m}$ polarization via confocal microscopy. However, shikonin-treated cells displayed a decrease in mitochondrial red fluorescence and elevated green fluorescence, signifying $\Delta_{\psi m}$ depolarization (Fig. 3A). The microscopic images were consistent with the flow cytometry analysis, confirming that shikonin destroyed $\Delta_{\psi \mathrm{m}}$ (Fig. 3B). Furthermore, we investigated the expression of proteins associated with mitochondria-mediated apoptosis. Shikonin enhanced the expression of the pro-apoptotic protein Bax and suppressed the expression of pro-survival/ anti-apoptotic protein $\mathrm{Bcl}-2$ (Fig. $3 \mathrm{C}$ ), leading to the translocation of cytochrome $\mathrm{c}$ from mitochondria into the cytosol (Fig. 3D). In addition, shikonin enhanced the expression of active (cleaved) caspase-9 and caspase-3 (Fig. 3E).

\section{Shikonin induces apoptosis via MAPK activation}

We next examined whether shikonin mediates apoptosis via the MAPK activation cascade. Western blotting data demonstrated that shikonin induced phosphorylation (activation) of JNK, p38, and ERK. Phosphorylation of JNK and p38 reached a maximum at $6 \mathrm{~h}$ and remained high after $24 \mathrm{~h}$, whereas phosphorylation of ERK peaked at $3 \mathrm{~h}$ and then decreased (Fig. 4A). Thus, ERK signaling demonstrated a faster and shorter reaction to shikonin stimulation than JNK and p38. We then checked whether the inhibitors (JNK, p38, and ERK inhibitors) suppress apoptosis induced by shikonin. Results of the MTT assay and Hoechst 33342 nuclear staining demonstrated the protective effect of SP600125 (JNK inhibitor) and SB203580 (p38 inhibitor) against shikonin-induced cell death (Fig. 4B, 4C). The effect of U0126 (ERK inhibitor) was weaker than the effects of other MAPK inhibitors, which was predicted because cell viability was detected at $24 \mathrm{~h}$, whereas ERK demonstrated maximum phosphorylation at the $3 \mathrm{~h}$ time point.

\section{Shikonin decreased EMT progression in radiation- resistant colon carcinoma cells}

Next, we explored the effect of shikonin on the migration of SNU-C5RR cells to understand its impact on EMT. First, we used a non-toxic concentration of shikonin, which allowed the observation of migrating cells up to $48 \mathrm{~h}$ after treatment. MTT data showed that $3 \mu \mathrm{M}$ of shikonin did not decrease the 
A

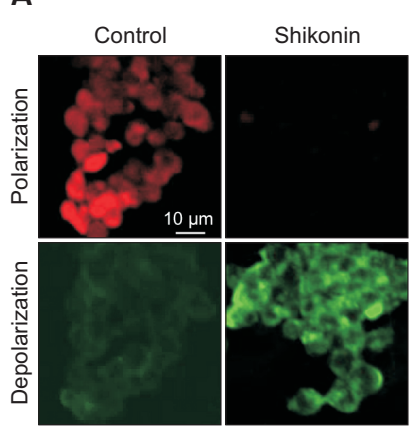

B
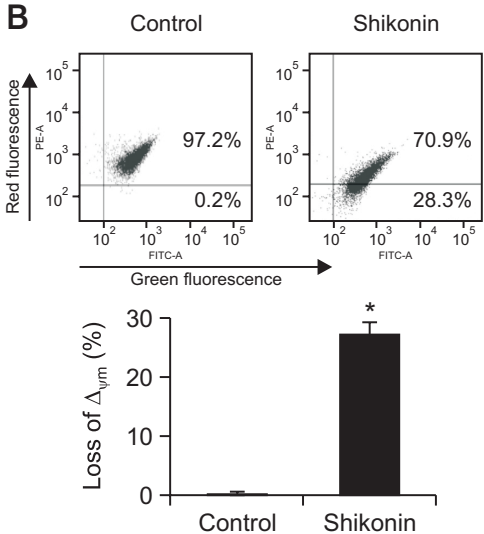

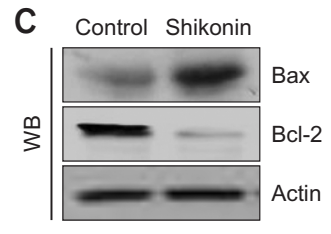

D Control Shikonin

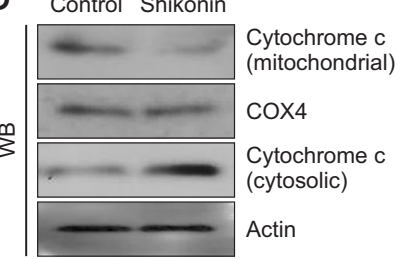

E Control Shikonin

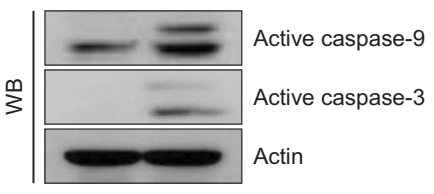

Fig. 3. Shikonin induces mitochondria-mediated apoptosis in SNU-C5RR colon cancer cells. Cells were stained with JC-1, and $\Delta_{\psi m}$ was detected via (A) confocal microscopy, and (B) flow cytometry. Cell lysates were subjected to western blotting for the following proteins, ${ }^{*} p<0.05$ vs control group; (C) Bax, and Bcl-2 protein levels were quantified, and actin was used as the loading control. (D) Mitochondrial cytochrome $\mathrm{C}$, and cytosolic cytochrome c levels were separately measured. COX4 and actin were used as loading controls for the mitochondrial and cytosolic fractions, respectively. (E) Active caspase-9, and active caspase-3 levels were verified via western blotting, and actin was used as the loading control.

A

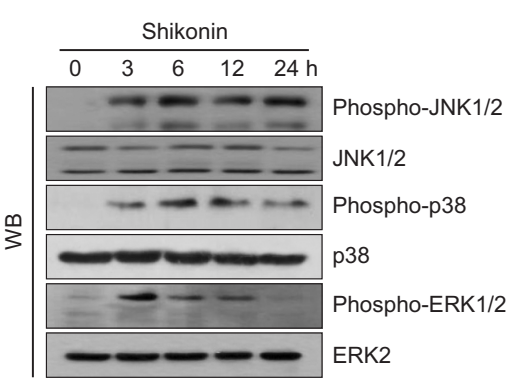

B

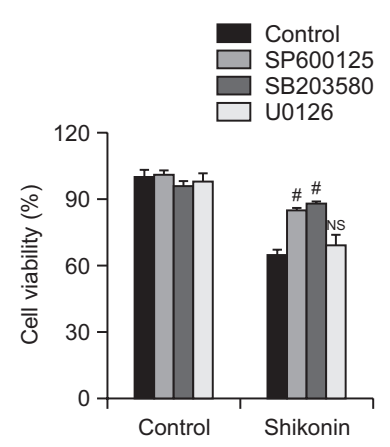

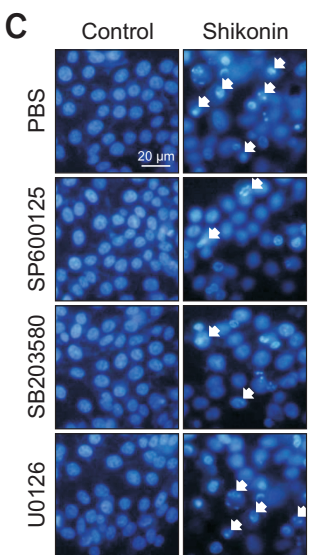

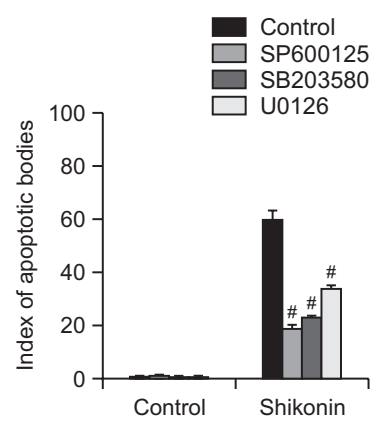

Fig. 4. Shikonin induces apoptosis via the MAPK signaling pathway in SNU-C5RR colon cancer cells. (A) Cells were harvested after $38 \mu M$ shikonin treatment at different time points $(0-24 \mathrm{~h})$ and subjected to western blotting using primary antibodies against phospho-JNK1/2, JNK1/2, phospho-p38, p38, phospho-ERK1/2, and ERK2. Following treatment with MAPK inhibitors and/or $38 \mu \mathrm{M}$ shikonin for $24 \mathrm{~h}$, (B) cell viability was assessed using the MTT assay and (C) apoptotic body formation was observed using Hoechst 33342 nuclear staining. Apoptotic bodies are indicated by arrows. ${ }^{*} p<0.05$ vs control cells; ${ }^{\#} p<0.05$ vs cells treated with shikonin only; NS, not significant compared to control. SP600125, JNK inhibitor; SB203580, p38 inhibitor; U0126, ERK inhibitor.

viability of SNU-C5RR cells $48 \mathrm{~h}$ after treatment (Fig. 5A); therefore, we used this concentration in subsequent studies of cellular mobility. We observed wounds established at the base of culture dishes 0 and $48 \mathrm{~h}$ after treatment with $3 \mu \mathrm{M}$ shikonin. While the gap was reduced in the control group, it was expanded in the shikonin-treated group (Fig. 5B). We then determined the expressions of $\mathrm{E}$ - and $\mathrm{N}$-cadherin, glycoproteins involved in initiating and stabilizing intercellular contacts. Expression of the epithelial marker E-cadherin and interstitial marker $\mathrm{N}$-cadherin was higher and lower, respectively, after shikonin treatment compared to that in untreated cells (Fig. 5C), confirming that shikonin attenuated EMT and thus the metastatic capacity of SNU-C5RR cells. Increased scavenging of superoxide anions and hydroxyl radicals by 3 $\mu \mathrm{M}$ shikonin was observed in the cell-free system (Fig. $5 \mathrm{D}$, $5 \mathrm{E})$. Furthermore, shikonin reduced cellular ROS production in SNU-C5RR cells, detected using confocal microscopy and flow cytometry after staining with DCF-DA (Fig. 5F, 5G).

\section{DISCUSSION}

Our investigation focused on radioresistance and ways to overcome it in colorectal cancer. We found that the herbal compound shikonin significantly suppressed the viability of radiation-resistant human colon carcinoma cells, and we attempted to identify an appropriate mechanism of apoptosis and EMT attenuation initiated by shikonin.

Apoptosis is a type of cell death that leads to several morphological and metabolic alterations, including chromatin condensation, nuclear fragmentation, and activation of special mediators (Wlodkowic et al., 2011). Thereby, the induction of 
A

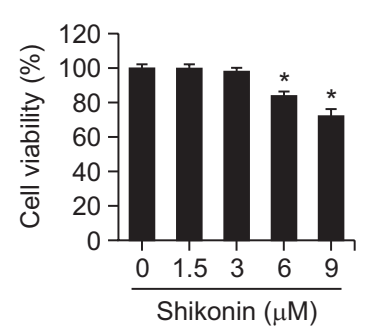

B

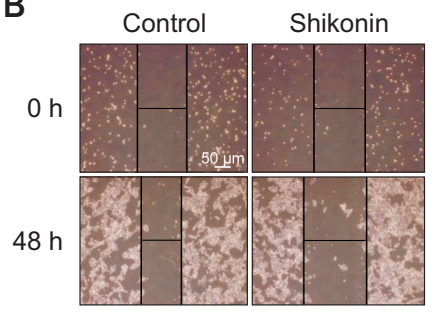

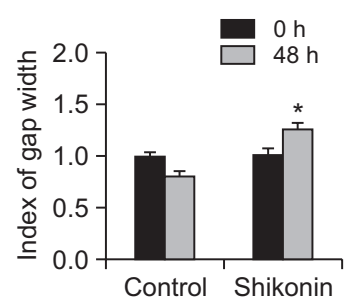

$\mathrm{E}$
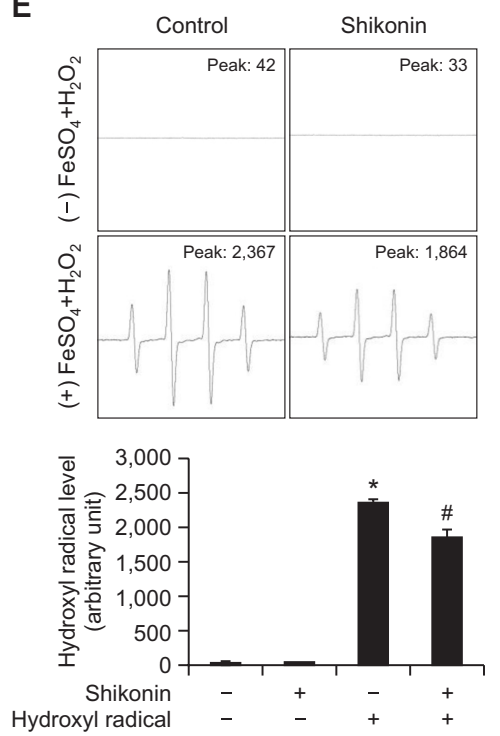

C

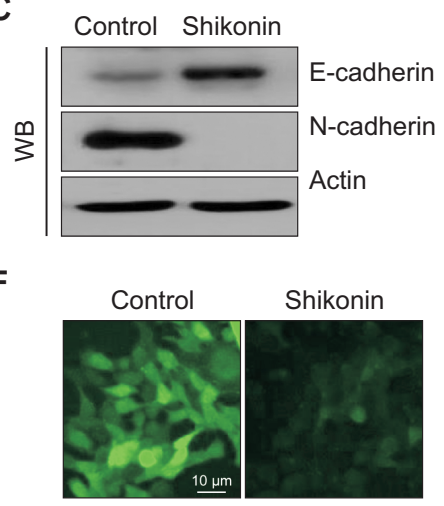

G
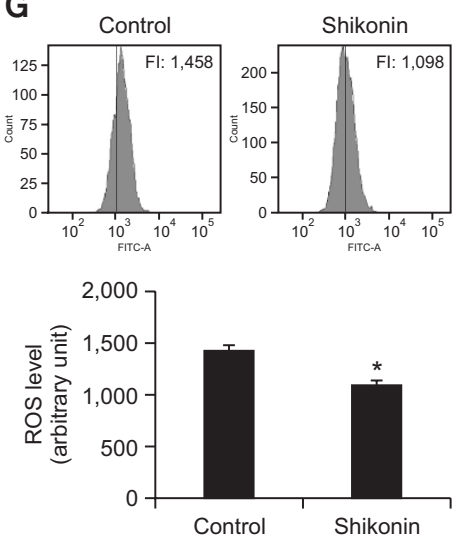

Superoxide anion

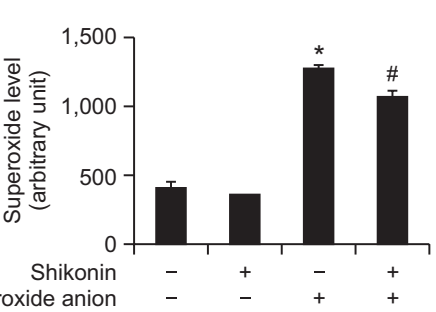

Fig. 5. Shikonin attenuated the epithelial-mesenchymal transition in SNU-C5RR colon cancer cells. (A) SNU-C5RR cells were treated with shikonin at concentrations $0,1.5,3,6$, or $9 \mu \mathrm{M}$ for $48 \mathrm{~h}$, and cell viability was assessed using the MTT assay. ${ }^{*} p<0.05$ vs control cells. (B) Cells were seeded in $100 \mathrm{~mm}$ culture dishes, and following treatment with $3 \mu \mathrm{M}$ shikonin, a scratch was established along the base of the dish. The wounded area was observed following treatment with or without $3 \mu \mathrm{M}$ shikonin after 0 and $48 \mathrm{~h}$. The gap was measured, and all data were quantified. Representative images for $48 \mathrm{~h}$ incubation are shown. ${ }^{*} p<0.05$ vs control cells. (C) Cell lysates were electrophoresed and used for western blotting with anti-E-cadherin and anti-N-cadherin antibodies. Actin was used as the loading control. The ability of $3 \mu \mathrm{M}$ shikonin to scavenge ROS in cell-free conditions was evaluated using (D) the xanthine/xanthine oxidase system for superoxide anion and (E) Fenton reaction $\left(\mathrm{FeSO}_{4}+\mathrm{H}_{2} \mathrm{O}_{2}\right)$ system for hydroxyl radical. ${ }^{*} p<0.05$ vs control, ${ }^{*} p<0.05$ vs radical only. SNU-C5RR cells were dyed with DCF-DA, and the fluorescence intensity was detected via (F) confocal microscopy, and (G) flow cytometry. * $p<0.05$ vs control.

apoptosis is an important strategy for anticancer therapy. Accumulating evidence shows that various apoptotic agents induce single- and double-strand DNA breaks (Lim et al., 2011). Our experiments indicated that the apoptotic body indices and the number of TUNEL-positive cells were elevated in shikonin-treated cells, as well as DNA single- and double-strand breaks, detected via comet assay. These results showed that the cytotoxic effect of shikonin is mediated through the apoptotic mechanism.

Numerous factors induce apoptosis via mitochondria-related pathways (Xiong et al., 2014). Oxidative stress or similar stress inductors destroy $\Delta_{\psi m}$, resulting in the outflow of cytochrome $\mathrm{c}$ from mitochondria into the cytosol. These events are controlled by pro-survival/anti-apoptotic $\mathrm{Bcl}-2$ and the proapoptotic protein Bax, leading to apoptosome complex formation. This complex initiates the caspase cascade and induces apoptosis (Yang et al., 2017). Thus, the typical markers of mitochondria-related apoptotic cell death include upregulation of Bax, downregulation of $\mathrm{Bcl}-2$, mitochondrial membrane depolarization, the release of cytochrome $\mathrm{c}$ from mitochondria, apoptotic body formation, and activation of the caspase cascade (Wang et al., 2016). Here, we detected the loss of $\Delta_{\psi m}$ following shikonin treatment. Moreover, $\mathrm{Bcl}-2$ expression was suppressed, whereas Bax expression increased. In addition, we observed the leakage of cytochrome $\mathrm{c}$ from mitochondria into the cytosol and the activation of caspase- 9 and caspase-3. These data provide evidence of mitochondria-related apoptosis induced by shikonin.

Many recent investigations have demonstrated that the cytotoxic properties of most compounds with anticancer activities are recognized through the activation of MAPK family members (Sun et al., 2015). Here, we focused on the role of MAPKs in apoptotic cell death induced by shikonin. The expression of activated JNK, p38, and ERK was enhanced with time upon treatment with shikonin, whereas the specific MAPK inhibitors demonstrated a cytoprotective effect against shikonin-induced cell death. Thus, our findings confirm that shikonin induces apoptotic cell death in SNU-C5RR cells via the activation of MAPK cascades.

EMT is closely related to tumor metastasis (Todosi et al., 
2012; Zhu et al., 2013; Ameli and Khalily, 2016; Matějka et al., 2017). This process is marked by the downregulation of epithelial markers (E-cadherin and $\beta$-catenin) and upregulation of mesenchymal markers (N-cadherin and fibronectin). Changes in $\mathrm{N}$-cadherin expression, as well as the EMT Ecadherin/N-cadherin switch, are known as independent risk factors for cancer growth and metastasis (Di Domenico et al., 2011). These findings have been fully explored in gastric, prostate, and oral carcinoma cell lines (Di Domenico et al., 2011; Feng et al., 2017; Ramamurthy et al., 2018), and there are many reports on EMT in colon cancer (Todosi et al., 2012; Zhu et al., 2013; Matějka et al., 2017). Here, the attenuation of migration capacity of SNU-C5RR cells was observed in the shikonin-treated group, implying that the occurrence of EMT destroys cell polarity, induces cell dedifferentiation, changes connections between cells, and improves the invasive ability. Expression of E-cadherin (an epithelial marker) was increased and expression of $\mathrm{N}$-cadherin (an interstitial marker) was suppressed in the shikonin-treated group, indicating that shikonin inhibits EMT. Excessive ROS production can promote cell invasion and migration, thereby promoting the acquisition of EMT characteristics in cancer cells (Kamiya et al., 2016; Ahn et al., 2017). ROS induce EMT by activating Snail expression and inhibits E-cadherin expression in MCF-7 cells (Lee et al., 2019), as well as mediate EMT and cell invasive potential in colon cancer (Jiao et al., 2016; Kang et al., 2018). ROS also act as a key signaling molecule mediating the fractionated ionizing radiation-induced EMT response through the activation of Akt/Src/Erk pathways (Kim et al., 2020).

Thus, we suggest that the EMT-attenuating property of shikonin may be related to its antioxidant effect. Shikonin significantly decreased ROS levels in SNU-C5RR cells, exerting anti-EMT effects, as increased ROS levels are important for EMT (Wang et al., 2010; Cichon and Radisky, 2014).

Taken together, these results suggest that SNU-C5RR colon cancer cells are sensitive to shikonin and that shikonin activates apoptosis and attenuates EMT. Thus, shikonin can be considered a potential candidate to overcome radiation resistance in colon cancer.

\section{CONFLICT OF INTEREST}

The authors have no conflicts of interest to declare.

\section{ACKNOWLEDGMENTS}

This work was supported by the research grant of Jeju National University in 2021.

\section{REFERENCES}

Ahn, H. M., Yoo, J. W., Lee, S., Lee, H. J., Lee, H. S. and Lee, D. S. (2017) Peroxiredoxin 5 promotes the epithelial-mesenchymal transition in colon cancer. Biochem. Biophys. Res. Commun. 487, 580-586.

Ameli, M. and Khalily, F. (2016) Analysis of colorectal cancer and polyp for presence herpes simplex virus and cytomegalovirus DNA sequences by polymerase chain reaction. J. Anal. Res. Clin. Med. 4, 82-89.

Andújar, I., Ríos, J. L., Giner, R. M. and Recio, M. C. (2013) Phar- macological properties of shikonin - a review of literature since 2002. Planta Med. 79, 1685-1697.

Baskar, R., Lee, K. A., Yeo, R. and Yeoh, K. W. (2012) Cancer and radiation therapy: current advances and future directions. Int. J. Med. Sci. 9,193.

Chae, S., Piao, M. J., Kang, K. A., Zhang, R., Kim, K. C., Youn, U. J., Nam, K. W., Lee, J. H. and Hyun, J. W. (2011) Inhibition of matrix metalloproteinase-1 induced by oxidative stress in human keratinocytes by mangiferin isolated from Anemarrhena asphodeloides. Biosci. Biotechnol. Biochem. 75, 2321-2325.

Cichon, M. A. and Radisky, D. C. (2014) ROS-induced epithelial-mesenchymal transition in mammary epithelial cells is mediated by NFkB-dependent activation of Snail. Oncotarget 5, 2827-2838.

Di Domenico, M., Pierantoni, G. M., Feola, A., Esposito, F., Laino, L., DE Rosa, A., Rullo, R., Mazzotta, M., Martano, M., Sanguedolce, F., Perillo, L., D’Angelo, L., Papagerakis, S., Tortorella, S., Bufo, P., Lo Muzio, L., Pannone, G. and Santoro, A. (2011) Prognostic significance of $\mathrm{N}$-Cadherin expression in oral squamous cell carcinoma. Anticancer Res. 31, 4211-4218.

Feng, L. M., Wang, X. F. and Huang, Q. X. (2017) Thymoquinone induces cytotoxicity and reprogramming of EMT in gastric cancer cells by targeting PI3K/Akt/mTOR pathway. J. Biosci. 42, 547-554.

Han, C. T., Kim, M. J., Moon, S. H., Jeon, Y. R., Hwang, J. S., Nam, C., Park, C. W., Lee, S. H., Na, J. B., Park, C. S., Park, H. W., Lee, J. M., Jang, H. S., Park, S. H., Han, K. G., Choi, Y. W., Lee, H. Y. and Kang, J. K. (2015) Acute and 28-day subacute toxicity studies of hexane extracts of the roots of Lithospermum erythrorhizon in Sprague-Dawley rats. Toxicol. Res. 31, 403-414.

He, G., He, G., Zhou, R., Pi, Z., Zhu, T., Jiang, L. and Xie, Y. (2016) Enhancement of cisplatin-induced colon cancer cells apoptosis by shikonin, a natural inducer of ROS in vitro and in vivo. Biochem. Biophys. Res. Commun. 469, 1075-1082.

Jeung, Y. J., Kim, H. G., Ahn, J., Lee, H. J., Lee, S. B., Won, M., Jung, C. R., Im, J. Y., Kim, B. K., Park, S. K., Son, M. J. and Chung, K. S. (2016) Shikonin induces apoptosis of lung cancer cells via activation of FOXO3a/EGR1/SIRT1 signaling antagonized by p300. Biochim. Biophys. Acta 1863, 2584-2593.

Jiao, L., Li, D. D., Yang, C. L., Peng, R. Q., Guo, Y. Q., Zhang, X. S. and Zhu, X. F. (2016) Reactive oxygen species mediate oxaliplatininduced epithelial-mesenchymal transition and invasive potential in colon cancer. Tumour Biol. 37, 8413-8423.

Jin, H., Gao, S., Guo, H., Ren, S., Ji, F., Liu, Z. and Chen, X. (2016) Re-sensitization of radiation resistant colorectal cancer cells to radiation through inhibition of AMPK pathway. Oncol. Lett. 11, 31973201.

Kamiya, T., Goto, A., Kurokawa, E., Hara, H. and Adachi, T. (2016) Cross talk mechanism among EMT, ROS, and histone acetylation in phorbol ester-treated human breast cancer MCF-7 cells. Oxid. Med. Cell. Longev. 2016, 1284372.

Kang, K. A., Ryu, Y. S., Piao, M. J., Shilnikova, K., Kang, H. K., Yi, J. M., Boulanger, M., Paolillo, R., Bossis, G., Yoon, S. Y., Kim, S. B. and Hyun, J. W. (2018) DUOX2-mediated production of reactive oxygen species induces epithelial mesenchymal transition in 5-fluorouracil resistant human colon cancer cells. Redox Biol. 17, 224-235

Khil, H., Kim, S. M., Hong, S., Gil, H. M., Cheon, E., Lee, D. H., Kim, Y. A. and Keum, N. (2021) Time trends of colorectal cancer incidence and associated lifestyle factors in South Korea. Sci. Rep. 11, 2413.

Kim, S., Kim, S. H. and Lee, C. E. (2020) SOCS1 represses fractionated ionizing radiation-induced EMT signaling pathways through the counter-regulation of ROS-scavenging and ROS-generating systems. Cell. Physiol. Biochem. 54, 1026-1040.

Ko, H., Kim, S. J., Shim, S. H., Chang, H. and Ha, C. H. (2016) Shikonin induces apoptotic cell death via regulation of p53 and Nrf2 in AGS human stomach carcinoma cells. Biomol. Ther. (Seoul) 24, 501-509.

Lee, S. Y., Ju, M. K., Jeon, H. M., Lee, Y. J., Kim, C. H., Park, H. G., Han, S. I. and Kang, H. S. (2019) Reactive oxygen species induce epithelial-mesenchymal transition, glycolytic switch, and mitochondrial repression through the DIx-2/Snail signaling pathways in MCF-7 cells. Mol. Med. Rep. 20, 2339-2346.

Lim, S. W., Ting, K. N., Bradshaw, T. D., Zeenathul, N. A., Wiart, C., 
Khoo, T. J., Lim, K. H. and Loh, H. S. (2011) Acalypha wilkesiana extracts induce apoptosis by causing single strand and double strand DNA breaks. J. Ethnopharmacol. 138,616-623.

Lu, D., Qian, J., Li, W., Feng, Q., Pan, S. and Zhang, S. (2015) $\beta$-Hydroxyisovaleryl-shikonin induces human cervical cancer cell apoptosis via PI3K/AKT/mTOR signaling. Oncol. Lett. 10, 34343442.

Matějka, V. M., Fínek, J. and Králíčková, M. (2017) Epithelial-mesenchymal transition in tumor tissue and its role for metastatic spread of cancer. Klin. Onkol. 30, 20-27.

Ramamurthy, V. P., Ramalingam, S., Gediya, L. K. and Njar, V. (2018) The retinamide VNLG-152 inhibits f-AR/AR-V7 and MNK-elF4E signaling pathways to suppress EMT and castration-resistant prostate cancer xenograft growth. FEBS J. 285, 1051-1063.

Shilnikova, K., Piao, M. J., Kang, K. A., Ryu, Y. S., Park, J. E., Hyun, Y. J., Zhen, A. X., Jeong, Y. J., Jung, U., Kim, I. G. and Hyun, J. W. (2018) Shikonin induces mitochondria-mediated apoptosis and attenuates epithelial-mesenchymal transition in cisplatin-resistant human ovarian cancer cells. Oncol. Lett. 15, 5417-5424.

Sun, Y., Liu, W. Z., Liu, T., Feng, X., Yang, N. and Zhou, H. F. (2015) Signaling pathway of MAPK/ERK in cell proliferation, differentiation, migration, senescence and apoptosis. J. Recept. Signal Transduct. Res. 35, 600-604.

Todosi, A. M., Gavrilescu, M. M., Aniţei, G. M., Filip, B. and Scripcariu, V. (2012) Colon cancer at the molecular level--usefulness of epithelial-mesenchymal transition analysis. Rev. Med. Chir. Soc. Med. Nat. lasi. 116, 1106-1111.

Trivedi, R., Müller, G. A., Rathore, M. S., Mishra, D. P. and Dihazi, H. (2016) Anti-leukemic activity of shikonin: role of ERP57 in shikonin induced apoptosis in acute myeloid leukemia. Cell. Physiol. Biochem. 39, 604-616.

Voon, D. C., Huang, R. Y., Jackson, R. A. and Thiery, J. P. (2017) The EMT spectrum and therapeutic opportunities. Mol. Oncol. 11, 878-891.

Vu, T. and Datta, P. K. (2017) Regulation of EMT in colorectal cancer: a culprit in metastasis. Cancers (Basel) $\mathbf{9}, 171$.

Wang, Q., Zhang, L., Yuan, X., Ou, Y., Zhu, X., Cheng, Z., Zhang, P., Wu, X., Meng, Y. and Zhang, L. (2016) The relationship between the $\mathrm{Bcl}-2 / \mathrm{Bax}$ proteins and the mitochondria-mediated apoptosis pathway in the differentiation of adipose-derived stromal cells into neurons. PLoS ONE 11, e0163327.

Wang, Z., Li, Y. and Sarkar, F. H. (2010) Signaling mechanism(s) of reactive oxygen species in epithelial-mesenchymal transition reminiscent of cancer stem cells in tumor progression. Curr. Stem Cell Res. Ther. 5, 74-80.

Wei, Y., Li, M., Cui, S., Wang, D., Zhang, C. Y., Zen, K. and Li, L. (2016) Shikonin inhibits the proliferation of human breast cancer cells by reducing tumor-derived exosomes. Molecules 21, 777.

Wlodkowic, D., Telford, W., Skommer, J. and Darzynkiewicz, Z. (2011) Apoptosis and beyond: cytometry in studies of programmed cell death. Methods Cell Biol. 103, 55-98.

Wong, M. C. S., Huang, J., Lok, V., Wang, J., Fung, F., Ding, H. and Zheng, Z. J. (2021) Differences in incidence and mortality trends of colorectal cancer worldwide based on sex, age, and anatomic location. Clin. Gastroenterol. Hepatol. 19, 955-966.

Wu, H., Sun, P., Feng, H., Zhou, H., Wang, R., Liang, Y., Lu, J., Zhu, W., Zhang, J. and Fang, J. (2012) Reactive oxygen species in a non-thermal plasma microjet and water system: generation, conversion, and contributions to bacteria inactivation-an analysis by electron spin resonance spectroscopy. Plasma Process. Polym. 9, 417-424.

Xiong, S., Mu, T., Wang, G. and Jiang, X. (2014) Mitochondria-mediated apoptosis in mammals. Protein Cell 5, 737-749.

Yang, Y., Zong, M., Xu, W., Zhang, Y., Wang, B., Yang, M. and Tao, L. (2017) Natural pyrethrins induces apoptosis in human hepatocyte cells via Bax-and Bcl-2-mediated mitochondrial pathway. Chem. Biol. Interact. 262, 38-45.

Zhu, Q. C., Gao, R. Y., Wu, W. and Qin, H. L. (2013) Epithelial-mesenchymal transition and its role in the pathogenesis of colorectal cancer. Asian Pac. J. Cancer Prev. 14, 2689-2698. 